\title{
BRYOFLORISTICAL DATA FROM AUSTRIAN PART OF SOPRON HILLS (ÖDENBURGER-GEBIRGE, E-AUSTRIA)
}

\author{
Péter Szúcs ${ }^{1 *}$ \& Harald Zechmeister ${ }^{2}$ \\ ${ }^{1}$ Eszterházy Károly University, Institute of Biology, Department of Botany and Plant \\ Physiology, H-3300 Eger, Leányka u. 6, Hungary; \\ 2University of Vienna, Department of Botany and Biodiversity Research, \\ Rennweg 14, 1030 Wien, Austria; *E-mail: szucs.peter@uni-eszterhazy.hu
}

\begin{abstract}
The Austrian part of Sopron Hills was a neglected area from the point of view of bryophyte floristics. In the investigated area 141 bryophytes were found, out of these 21 liverworts and 120 mosses. There were nine species new to Burgenland: Jungermannia gracillima, Pellia neesiana, Campylopus pyriformis, Leucobryum juniperoideum, Orthotrichum lyellii, Pohlia elongata, Pohlia prolifera, Pseudephemerum nitidum, Trichodon cylindricus.

Some species were detected from the territory which are otherwise rare in Austria, such as: Fossombronia wondraczekii, Riccardia palmata, Brachythecium mildeanum, Didymodon cordatus, Didymodon vinealis, Fissidens adianthoides, Microbryum davallianum, Plagiomnium elatum, Pleuridium acuminatum, Pleuridium subulatum, Pottia intermedia, Pseudephemerum nitidum.

13 species are listed as threatened in the Red Data List of bryophytes in Lower Austria, and so do 11 species in the overall Austrian Red Data list of bryophytes. No species of the Annex II of the FFH-directive were found.
\end{abstract}

Keywords: bryophytes, rare species, red-list status, Burgenland

\section{INTRODUCTION}

To date no significant bryological research has been carried out at the Austrian part of the Sopron Hills, leaving this territory almost unexplored. The overall Burgenland itself has only a very marginal recent bryological examination. The research of Latzel (1941) involved only the Hungarian parts of the Lower Alps. The work of Maurer (1965) on the moss species of the Burgenland presents field data for the Southern part of the Burgenland. Schlüsslmayr (2001) examined the Leithagebirge in northern Burgenland and Zechmeister $(2004,2005 a)$ studied the bryophyte flora of the 
Seewinkel. Another research of Zechmeister (2008) involved the peatmoss habitats of the Burgenland. Within the region of these studies only the work of Zechmeister (2005b) describes the serpentine outcrops in the area. Preliminary results for the Sopron Hills have first been published by Szúcs and Szmorad (2009). No checklist or Red Data List can be found for the Burgenland. The Red Data List of Lower Austria, which was based on abundant latest floristical data, has been published only recently (Zechmeister et al. 2013). Nevertheless, the Sopron Hills are located beyond the boundaries of the Lower Austria region, which makes the relation and direct comparison of the obtained data difficult.

The proposed article intends to fill this gap in knowledge and provides the latest bryofloristical results for the Sopron Hills.

\section{Study area}

The Sopron Hills are the north-eastern and relatively low height (between 600 and $800 \mathrm{~m}$ a.s.l.) subrange of the Alps. To the west they are separated from the adjacent, nearly north-south running Rosalia Hills by a saddle above the village of Sieggraben (Szmorad 2011).

In aspect of geology the western part of the hills towards the Rosalia Hills is covered with miocene sandy, gravel-clay sediments. In the southern part of the hills (between Ritzing and Neckenmarkt, also south of Kalkgruben) there are penetrations of Leitha limestone, and acidic sandstones located between Neckenmarkt and Harka. In the north-western part of the region, large-scale areas are covered with a Badenian clay formation (Szmorad 2011). The most frequent soil types of the territory are brown forest soils. The hydrographic network of the area consists of small streams. The eastern hill front is warmer and dryer, the western, inner area is much cooler and has higher precipitation. The mean annual temperature varies between 8-9 ${ }^{\circ} \mathrm{C}$, and the annual precipitation usually between $650-900 \mathrm{~mm}$ (Király 2004, Szmorad 2011).

From the phytogeographical point of view the Sopron Hills are situated in the border region of the Eastern Alpine (Noricum) and the Pannonian (Pannonicum) floristic regions. In the hills the mesophilous and acidophilous decidous forests are dominant, but there are large-scale areas of secondary coniferous forest stands (Király 2004, Szmorad 2010). 


\section{MATERIALS AND METHODS}

The fieldwork has been carried out between 2009 and 2014. The main habitats, such as acidophylous deciduous and mixed forests, planted coniferous forests, wetlands, grasslands, streams, forest roads, lakes, quarries and buildings were investigated based on a subjective site selection. Bryophytes were collected from all available substrates (soil, concrete, tree bark and root, decayed wood, stone and plastic foil).

The nomenclature follows Köckinger et al. (2015). The species names are given, as well as the Red Data List status which was given to these species for Lower Austria by Zechmeister et al. (2013), number of sampling site(s) and the substrate within. Specimens have been deposited in bryophyte collections of the Eszterházy Károly University in Eger (EGR) and in the private herbarium of Harald Zechmeister at University of Vienna. The site details are listed in the appendix.

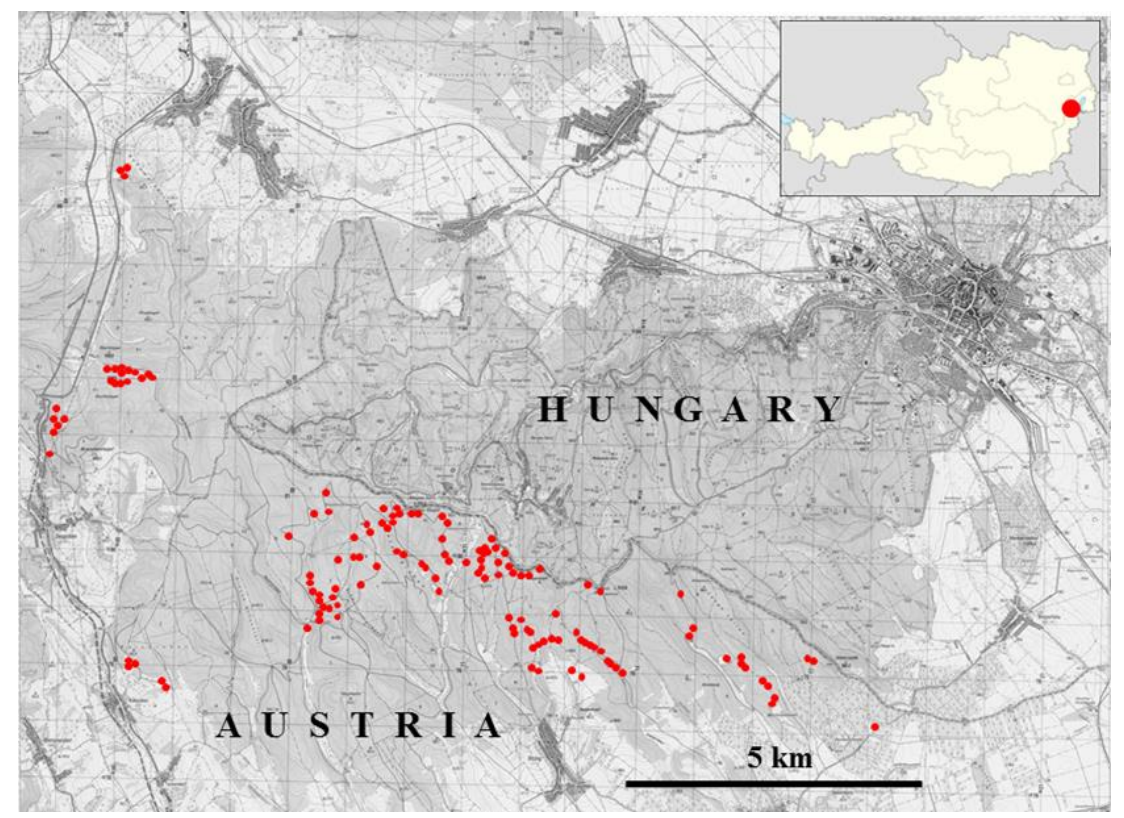

Figure 1. The sampling sites in the investigated area 


\section{RESULTS}

\section{List of species}

In total 141 species were found, out of these were 120 bryophytes and 21 liverworts.

Hepaticeae

Blasia pusilla L. - LC - 79, 80: on soil

Calypogeia fissa (L.) Raddi - LC - 101: on soil

Cephalozia bicuspidata (L.) Dumort. - LC - 98, 101: on soil

Cephaloziella divaricata (Sm.) Schiffn. - LC - 7, 38, 62, 66, 63, 90: on soil

Conocephalum conicum (L.) Dumort. - LC - 46: on soil

Fossombronia wondraczekii (Corda) Lindb. - NT - 79: on soil

Frullania dilatata (L.) Dumort. - LC - 13: bark of Fagus sylvatica; 86: bark of Malus sylvestris

Jungermannia gracillima Sm. - LC - 63, 66: on soil

Lophocolea bidentata (L.) Dumort. - LC - 75, 67, 84: on soil

Lophocolea heterophylla (Schrad.) Dumort. - LC - 14: root of Alnus glutinosa; 16: on bark; 15, 78: on decayed wood; 90 : on soil; 108: on rooted Picea abies trunk

Lophocolea minor Nees - LC - 33: on soil

Metzgeria furcata (L.) Dumort. - LC - 108: on bark of Tilia; 119: on bark of Fagus sylvatica

Nowellia curvifolia (Dicks.) Mitt. - LC - 108: on rooted Picea abies trunk

Pellia endiviifolia (Dicks.) Dumort. - LC - 4, 79: on soil

Pellia neesiana (Gottsche) Limpr. - LC - 30: on soil

Plagiochila asplenoides (L. emend. Taylor) Dumort. - LC - 46, 108: on soil

Plagiochila porelloides (Torr. ex Nees) Lindenb. - LC - 70, 84: on soil

Ptilidium pulcherrimum (Weber) Vain. - LC - 90: on soil; 91: bark of Quercus petraea

Radula complanata (L.) Dumort. - LC - 19: bark of Acer campestre; 46: bark of Acer pseudoplatanus and Alnus glutinosa; 78: bark of Corylus avellana; 119: bark of Fagus sylvatica

Riccardia palmata (Hedw.) Carruth. - VU - 108: on rooted Picea abies trunk

Scapania nemorea (L.) Grolle - LC - 101: on soil 
Musci

Abietinella abietina (L. ex Hedw.) M.Fleisch. var. abietina - LC - 87: on soil

Amblystegium riparium (L. ex Hedw.) Schimp. - LC - 16: on bark and root; 77: on piece of wood

Amblystegium serpens (L. ex Hedw.) Schimp. - LC - 19: bark of Acer campestre; 21, 71: on concrete; 74: bark of Alnus glutinosa, bark of Sambucus nigra; 78: bark of Corylus avellana

Anomodon attenuatus (Hedw.) Huebener - LC - 116: on limestone Anomodon viticulosus (Hedw.) Hook. \& Taylor - LC - 116: on limestone

Atrichum undulatum (Hedw.) P.Beauv. - LC - 2, 3, 8, 10, 12, 14, 62, 63, 90: on soil

Barbula convoluta Hedw. - LC - 5: on soil

Barbula unguiculata Hedw. - LC - 44, 77, 89: on soil

Bartramia pomiformis Hedw. - LC - 108: on soil

Brachytheciastrum velutinum (L. ex Hedw.) Ignatov \& Huttunen LC - 1, 14, 16: on decayed wood and soil; 7, 33, 46: on soil; 74 : bark of Alnus glutinosa

Brachythecium albicans (Neck. ex Hedw.) Schimp. - LC - 39, 42, 87: on soil

Brachythecium glaerosum (Bruch ex Spruce) Schimp. var. glareosum - LC - 3, 10, 11, 87: on soil

Brachythecium mildeanum (Schimp.) Schimp. - EN - 109: on soil

Brachythecium rivulare Schimp. - LC - 16: on root; 50: stump of Alnus glutinosa; 75, 76, 89: on soil; 78, 106: on decayed wood

Brachythecium rutabulum (L. ex Hedw.) Schimp. - LC - 16: on bark; 78: on decayed trunk, on concrete; 4, 39, 42, 77: on soil; 83: on decayed wood

Brachythecium salebrosum (Hoffm. ex F.Weber \& D.Mohr) Schimp.

- LC - 7: on soil; 14, 74: bark of Alnus glutinosa, bark of Sambucus nigra

Bryum argenteum Hedw. - LC - 116: on soil

Bryum capillare Hedw. - LC - 104: on soil

Bryum elegans Nees var. elegans - LC - 73: on soil

Bryum moravicum Podp. - LC - 47: bark of Fraxinus sp.; 119: bark of Fagus sylvatica

Bryum rubens Mitt. - LC - 62: on soil 
Calliergonella cuspidata (L. ex Hedw.) Loeske - LC - 3, 20, 55, 56, 63, 75: on soil; 71: on plastic foil; 78: on concrete, on decayed wood

Campylopus pyriformis (Schultz) Brid. - VU - 53: on soil

Ceratodon purpureus (Hedw.) Brid. - LC - 2, 6, 7, 10, 11, 13, 39, 42, $48,60,62,87,90$ : on soil

Cirriphyllum crassinervium (Taylor) Loeske \& M.Fleisch. - LC 116: on limestone

Cirriphyllum piliferum (Schreb. ex Hedw.) Grout - LC - 18, 39, 42, 48, 63, 77: on soil

Climacium dendroides (Hedw.) F.Weber \& D.Mohr - LC - 18: on soil Cratoneuron filicinum (L. ex Hedw.) Spruce - LC - 37, 50, 56, 77, 78: on soil

Dicranella heteromalla (Hedw.) Schimp. - LC - 2, 7, 10, 12, 13, 62, 63, 68, 79, 90: on soil

Dicranella staphylina H.Whitehouse - LC - 39: on soil

Dicranella varia (Hedw.) Schimp. - LC - 77: on soil

Dicranum montanum Hedw. - LC - 10, 12, 15: on decayed wood; 81: on decayed log; 91: bark of Quercus petraea; 91: on soil

Dicranum polysetum Sw. ex anon. - LC - 62: on soil

Dicranum scoparium Hedw. - LC - 6: on decayed wood; 13, 38, 42, 90, 91: on soil; 82: stump of Alnus glutinosa

Didymodon cordatus Jur. - NT - 111: on soil; 115: surface of calcareous building; 116: on limestone

Didymodon rigidulus Hedw. - LC - 115: surface of calcareous building; 116: on limestone

Didymodon vinealis (Brid.) R.H.Zander - EN - 115: surface of calcareous building

Drepanocladus aduncus (Hedw.) Warnst. - LC - 77: on soil

Encalypta streptocarpa Hedw. - LC - 116: on limestone

Eurhynchiastrum pulchellum (Hedw.) Ignatov \& Huttunen var. pulchellum - LC - 70: on soil

Eurhynchium angustirete (Broth.) T.J.Kop. - LC - 14: decayed wood; $39,42,63,67,68,73,84,91$ : on soil

Fissidens adianthoides Hedw. - NT - 57: on soil

Fissidens bryoides Hedw. - LC - 73: on soil

Fissidens taxifolius Hedw. subsp. taxifolius - LC - 1: on soil

Funaria hygromertica Hedw. - LC - 62, 120: on soil

Grimmia pulvinata (Timm. ex Hedw.) Sm. - LC - 71: on concrete Herzogiella seligeri (Brid.) Z.Iwats. - LC - 6, 15: on decayed wood 
Heterocladium heteropterum (Brid.) Schimp. - LC - 43: on soil Homalia trichomanoides (Hedw.) Brid. - LC - 49: on root

Homalothecium lutescens (Hedw.) H.Rob. - LC - 3: on soil

Homalothecium philippeanum (Spruce) Schimp. - LC - 47: bark of Fraxinus sp.; 116: on limestone

Homomallium incurvatum (Schrad. ex Brid.) Loeske - LC - 21: on concrete; 116: on limestone

Hylocomnium splendens (Hedw.) Schimp. - LC - 18, 91: on soil

Hypnum cupressiforme Hedw. var. cupressiforme - LC - 1: on decayed wood and soil; 14: root of Alnus glutinosa; 47: bark of Fraxinus; 78: bark of Alnus glutinosa; 8, 38, 84, 90, 91: on soil; 90: bark of Malus sylvestris

Hypnum cupressiforme var. lacunosum Brid. - LC - 73: on soil

Hypnum lindbergii Mitt. - LC - 71: on plastic foil; 78: on decayed wood, 4, 39, 48, 84: on soil

Isothecium alopecuroides (Lam. ex Dubois) Isov. - LC - 119: root swelling of Fagus sylvatica

Leskea polycarpa Ehrh. ex Hedw. - LC - 19: bark of Acer campestre Leucobryum glaucum (Hedw.) Ångstr. - LC - 91: on soil

Leucobryum juniperoideum (Brid.) Müll.Hal. - LC - 90, 91: on soil

Leucodon sciuroides (Hedw.) Schwägr. - LC - 86: bark of Malus sylvestris

Microbryum davallianum (Sm.) R.H.Zander - EN - 44: on soil

Mnium hornum Hedw. - LC - 108: root swelling of Tilia sp.

Mnium marginatum (Dicks.) P.Beauv. - LC - 115: surface of calcareous building

Orthotrichum affine Schrad. ex Brid. - LC - 28: bark of Fraxinus sp.

Orthotrichum anomalum Hedw. - LC - 21: on concrete; 116: on limestone

Orthotrichum cupulatum Hoffm. ex Brid. var. cupulatum - LC 116: on limestone

Orthotrichum diaphanum Schrad. ex Brid. - LC - 65: bark of Sambucus nigra

Orthotrichum lyellii Hook. \& Taylor - LC - 28: on bark of Fraxinus sp.

Orthotrichum obtusifolium Brid. - LC - 25: on bark of Fraxinus sp.

Orthotrichum pallens Bruch ex Brid. - LC - 35: bark of Fagus sylvatica

Orthotrichum speciosum Nees - LC - 35: bark of Fagus sylvatica 
Oxyrrhynchium hians (Hedw.) Loeske var. hians - LC - 82: root swelling of Alnus glutinosa; 89: on soil

Palustriella commutata (Hedw.) Ochyra var. commutata - LC - 57, 109: on soil; 71: on plastic foil

Phascum cuspidatum Schreb. ex Hedw. var. cuspidatum - LC - 44, 45: on soil

Physcomitrium pyriforme (Hedw.) Bruch \& Schimp. - LC - 60: on soil

Plagiomnium affine (Blandow ex Funck) T.J.Kop. - LC - 78: on decayed trunk; $18,73,90$ : on soil

Plagiomnium cuspidatum (Hedw.) T.J.Kop. - LC - 16: on root; 18 : on soil; 78: on concrete

Plagiomnium elatum (Bruch \& Schimp.) T.J.Kop. - VU - 68: on soil

Plagiomnium rostratum (Schrad.) T.J.Kop - LC - 106: on decayed wood

Plagiomnium undulatum (Hedw.) T.J.Kop. - LC - 21, 68, 75, 78: on soil; 15: on decayed wood; 82: stump of Alnus glutinosa

Plagiothecium cavifolium (Brid.) Z.Iwats. - LC - 12, 63: on soil

Plagiothecium denticulatum (L. ex Hedw.) Schimp. - LC - 12: on soil; 15: on decayed wood

Plagiothecium laetum Schimp. var. laetum - LC - 12, 13, 16: on soil

Plagiothecium nemorale (Mitt.) A.Jaeger - LC - 12: on soil

Plagiothecium succulentum (Wilson) Lindb. - LC - 16, 63: on soil

Platygyrium repens (Brid.) Schimp. - LC - 78: bark of Alnus glutinosa

Pleuridium acuminatum Lindb. - VU - 2, 62: on soil

Pleuridium subulatum (Hedw.) Rabenh. - NT - 5, 63: on soil

Pleurozium schreberi (Willd. ex Brid.) Mitt. - LC - 18, 39, 42, 62, 84: on soil

Pogonatum aloides (Hedw.) P.Beauv. - LC - 62, 63: on soil

Pohlia elongata Hedw. var. elongata - LC - 12: on soil

Pohlia melanodon (Brid.) A.J.Shaw - LC - 1, 89: on soil

Pohlia nutans (Hedw.) Lindb. subsp. nutans - LC - 2, 6, 63, 90: on soil

Pohlia proligera (Kindb.) Lindb. ex Broth. - VU-R - 54: on soil

Pohlia wahlenbergii (F.Weber \& D.Mohr) A.L.Andrews var. wahlenbergii - LC - 5: on soil

Polytrichum formosum Hedw. - LC - 2, 6, 11, 13, 90: on soil

Polytrichum juniperinum Willd. ex Hedw. - LC - 48, 79, 90: on soil 
Polytrichum piliferum Schreb. ex Hedw. - LC - 8, 62, 70, 90, 111: on soil

Pottia intermedia (Turner) Fürnr. - VU - 39: on soil

Pottia truncata (Hedw.) Bruch \& Schimp. - LC - 35: on soil

Pseudephemerum nitidum (Hedw.) Loeske - EN - 98: on soil

Pseudoleskeella nervosa (Brid.) Nyholm - LC - 19: bark of Acer campestre

Pseudoscleropodium purum (L. ex Hedw.) M.Fleisch. - LC - 39, 42, 84, 90, 114: on soil

Pterigynandrum filiforme Hedw. var. filiforme - LC - 119: on bark of Fagus sylvatica

Pylaisia polyantha (Hedw.) Schimp. - LC - 19: bark of Acer campestre; 74: bark of Sambucus nigra; 78: bark of Corylus avellana

Racomitrium canescens (Timm. ex Hedw.) Brid. subsp. canescens - LC - 89: on soil

Rhizomnium punctatum (Hedw.) T.J.Kop. - LC - 16: on root and bark; 74: bark of Alnus glutinosa; 15, 83: on decayed wood; 46, 50, 82: stump of Alnus glutinosa

Rhyncostegium murale ((Neck. ex Hedw.) Schimp. - LC - 21: on concrete; 115: on calcareous building

Rhytidiadelphus squarrosus (L. ex Hedw.) Warnst. - LC - 18, 42, 84: on soil

Schistidium crassipilum H.H.Blom - LC - 21, 71: on concrete

Syntrichia ruralis (Hedw.) F.Weber \& D.Mohr - LC - 86: on concrete

Tetraphis pellucida Hedw. - LC - 16, 106, 113: on decayed wood

Thuidium assimile (Mitt.) A.Jaeger - LC - 39: on soil; 78: on decayed wood

Thuidium delicatulum (Hedw.) Schimp. - LC - 18, 38, 84: on soil

Thuidium tamariscinum (Hedw.) Schimp. - LC - 18, 78: on decayed wood; 42, 68: on soil

Trichodon cylindricus (Hedw.) Schimp. - LC - 1, 17: on soil

Tortula muralis Hedw. var. muralis - LC - 71: on concrete

Ulota bruchii Hornsch. ex Brid. - LC - 25, 110: bark of Fraxinus sp.

Ulota crispa (Hedw.) Brid. - LC - 17: bark of Betula pendula 


\section{DISCUSSION}

On the basis of these results, the bryophyte list of the project area was compared to the national (Grims and Köckinger 1999, Saukel and Köckinger 1999) and regional bryophyte Red Data List (Zechmeister et al. 2013) as well as to the Hungarian Red data List (Papp et al. 2010) (Table 1).

Table 1. The Red Data List status of bryophytes in Lower Austria (Niederösterreichs) (Zechmeister et al. 2013), Austria (Grims and Köckinger 1999, Saukel and Köckinger 1999) and Hungary (Papp et al. 2010).

Comments: Red Data List status in Austria:*VU (gefährdet), *reg. VU (r3 gefährdet, *EN (stark gefährdet) (Grims and Köckinger 1999, Saukel and Köckinger 1999).

\begin{tabular}{lccc}
\hline Species name & $\begin{array}{c}\text { Lower } \\
\text { Austria }\end{array}$ & Austria & Hungary \\
\hline Blasia pusilla (Hepaticeae) & LC & LC & EN \\
\hline Calypogeia fissa & LC & LC & NT \\
\hline Fossombronia wondraczekii & NT & VU* & DD \\
\hline Jungermannia gracillima & LC & LC & NT \\
\hline Nowellia curvifolia & LC & LC & VU \\
\hline Pellia neesiana & LC & LC & unknown \\
\hline Plagiochila asplenoides & LC & LC & NT \\
\hline Ptilidium pulcherrimum & LC & LC & NT \\
\hline Riccardia palmata & VU & LC & NT \\
\hline Scapania nemorea & LC & LC & VU \\
\hline Brachythecium mildeanum (Musci) & EN & VU* & LC-att \\
\hline Brachythecium glaerosum var. glareosum & LC & LC & NT \\
\hline Campylopus pyriformis & VU & EN* & DD \\
\hline Dicranella staphylina & NT & LC & NT \\
\hline Didymodon cordatus & NT & VU* & LC-att \\
\hline Didymodon vinealis & EN & VU* & LC \\
\hline Eurhynchiastrum pulchellum var. pulchellum & LC & LC & NT \\
\hline Fissidens adianthoides & NT & reg. VU* & NT \\
\hline Heterocladium heteropterum & LC & LC & unknown \\
\hline Microbryum davallianum & EN & VU & LC-att \\
\hline Mnium hornum & LC & LC & NT \\
\hline Orthotrichum cupulatum & LC & reg. VU* & LC-att \\
\hline Orthotrichum obtusifolium & LC & LC & NT \\
\hline Palustriella commutata var. commutata & LC & LC & EN \\
\hline Plagiomnium elatum & VU & VU & LC-att \\
\hline Plagiothecium succulentum & LC & VU & LC \\
\hline Pleuridium acuminatum & VU & LC & LC-att \\
\hline Pleuridium subulatum & NT & LC & LC-att \\
\hline Pohlia elongata var. elongata & LC & LC & DD \\
\hline Pohlia proligera & VU-R & LC & DD \\
\hline Pottia intermedia & VU & LC & LC-att \\
\hline Pseudephemerum nitidum & EN & VU & LC-att \\
\hline Ulota bruchii & LC & LC & VU \\
\hline Ulota crispa & LC & LC & NT \\
\hline & & & \\
\hline
\end{tabular}


No species of the Annex II of the FFH-directive were found in the investigated area. However, Leucobryum sp. which is part of the Annex V of the respective directive was found several times (for details see the species list and corresponding site numbers).

The bryophyte flora of the investigated area resembles that of other lowlands in northern or southern Austria and despite its position in eastern Austria it has only a very small number of species typical or widespread in the Pannonian area (e.g. Didymodon vinealis). This is due to the fact that most habitats in the Sopron Hills do not represent typical eastern Austrian habitats as natural dry grasslands or vineyards. Furthermore, most of the sites are situated at comparable higher elevation as most of the sites in the east of Austria. In addition the investigated sites represent a wide range of woods and consequence microclimate and soils are more humid than at „typical” Pannonian sites.

Related to its bedrock the flora is dominated by acidophilous species and only a very few species related to calcareous bedrock were found. The large extent of woods in the area favours a wide range of shade tolerant plants, as well as epiphytes and species restricted to dead logs. Compared to other studies in the centre of the Pannonian area of the Burgenland (e.g. Zechmeister 2005b) which is slightly north of the study area, hepatics show a comparable high number in this study, which is mainly a result of the more humid situation in the investigated area.

The number of threatened species is low in our study as the investigated sites were situated within habitats which are neither threatened by human activity and nore rare in occurrence, too.

Acknowledgement - The project was financed by the „Ernst Mach-Stipendium der AÖU der Aktion Österreich-Ungarn” and "Stipendium der AÖU für Universitätslehrer der Aktion Österreich-Ungarn” scholarships.

The article was funded by „Társadalmi Megújulás Operatív Program Kutatás, Innováció, Együttmúködések - Társadalmi innováció és kutatási hálózatok együttműködésének erősítése az Eszterházy Károly Főiskola, a Bay Zoltán Alkalmazott Kutatási Nonprofit Kft. és az Agria TISZK Közhasznú Nonprofit Kft. együttmúködésével" called project (TÁMOP-4.2.1.D-15/1/KONV-2015-0013). The project was supported by the European Union and the European Social Fund cofunded. The authors are grateful to Ferenc Szmorad and Tamás Pócs for their help in literature access and interpretation. 


\section{REFERENCES}

GRIMS, F. \& KÖCKINGER, H. (1999). Rote Liste gefährdeter Laubmoose (Musci) Österreichs. In: NikLfELD, H. (ed.), Rote Liste gefährdeter Pflanzen Österreichs. Austria Medien Service, Wien, pp. 157-171.

KIRÁLY, G. (ed., 2004). A Soproni-hegység edényes flórája. (Die Gefäßpflanzenflora des Ödenburger Gebirges). Flora Pannonica 2(1): 7-12.

KöCKInGer, H., SCHRÖCK, C., KriSiaI, R. \& ZeChmeister, H.G. (2015). Checklist of Austrian bryophytes. http://131.130.59.133/projekte/moose/ [01.11.2015.]

LATZEL, A. (1941). Beitrag zur Kenntnis der Moose des Ostralpenrandgebiets. Beih. Bot. Centralbl. 61: 211-260.

Maurer, W. (1965). Die Moose des Südburgenlandes. Wiss. Arb. Burgenland 32: 540.

Papp, B., ERZberger, P., Ódor, P., Hock, Zs., Szövényi, P., Szurdoki, E. \& TóTh, Z. (2010). Updated checklist and redlist of Hungarian bryophytes. Studia botanica hungarica 41: 31-59.

SAUKEL, J. \& KöCKINGER, H. (1999). Rote Liste gefährdeter Lebermoose (Hepaticae) und Hornmoose (Anthocerotae) Österreichs. In: NiKLFELD, H. (ed.): Rote Liste gefährdeter Pflanzen Österreichs, Austria Medien Service, Wien, pp. 172-177.

SchlÜSSLMAYR, G. (2001). Die Moosvegetation des Leithagebirges im Burgenland. Verhandlungen der Zoologisch-Botanischen Gesellschaft in Wien 138: 65-93.

SzMorad, F. (2010). A Soproni-hegység erdeinek történeti, növényföldrajzi és cönológiai vizsgálata. (The historical, phytogeographical and coenological investigations on the forest of the Sopron Hills). PhD dissertation in manuscript, Pécsi Tudományegyetem Biológia Doktori Iskola, Pécs. 128 pp.

Szmorad, F. (2011). The Riparian Alder Forests of the Sopron Hills. Acta Silvatica \& Lignaria Hungarica 7: 109-124.

SzŰCS, P. \& SzMORAD, F. (2009). Ergänzungen zur Moosflora des Ödenburger Gebirges. Flora Pannonica 7: 61-72.

ZeChmeIsteR, H.G. (2004). Die Moosflora im Natura 2000 Gebiet "Neusiedlersee", unter besonderer Berücksichtigung der Salzwiesen im Seewinkel. Verhandlungen der Zoologisch-Botanischen Gesellschaft in Wien 141: 43-62.

ZeChmeister, H.G. (2005a). Bryophytes of continental salt meadows in Austria. Journal of Bryology 27: 297-302.

ZeChmeister, H.G. (2005b). Die Moosflora der Serpentinrasen im Burgenland. Verhandlungen der Zoologisch-Botanischen Gesellschaft in Wien 142: 9-15.

Zechmeister, H.G. (2008). Vorkommen und Gefährdung der Torfmoose (Gattung Sphagnum L.) im Burgenland. Verhandlungen der Zoologisch-Botanischen Gesellschaft in Wien 145: 97-106.

Zechmeister, H.G., Hagel, H., Gendo, A., Osvaldik, V., Patek, M., Prinz, M., Schröck, C. \& KöCKINGER, H. (2013). Die Rote Liste der Moose Niederösterreichs. Wissenschaftliche Mitteilungen Niederösterreichisches Landesmuseum 24: 4126.

(submitted: 30.11.2015, accepted: 04.01.2016) 


\section{APPENDIX}

\section{Site details}

1. Burgenland, Bezirk Oberpullendorf, Ritzing, Angerwald, near country border, planted Picea abies forest (21.10.2009) N47³8'24.7" E16 29'43.9”, 477 m. [8364.4]

2. Burgenland, Bezirk Oberpullendorf, Ritzing, Angerwald, near country border, Fagus

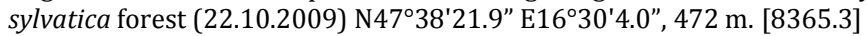

3. Burgenland, Bezirk Oberpullendorf, Ritzing, near village, on gravelly forest road (23.07.2013) N47³8'33.9” E 16²8'19.4", 430 m. [8364.4]

4. Burgenland, Bezirk Oberpullendorf, Ritzing, near village, next to gravelly forest road (23.07.2013) N47³8'34.9” E16²8'17.0", 433 m. [8364.4]

5. Burgenland, Bezirk Oberpullendorf, Ritzing, lake (23.07.2013) N47³8'45.5" E16²8'11.0", $436 \mathrm{~m}$. [8364.4]

6. Burgenland, Bezirk Oberpullendorf, Ritzing, on embankment of forest road (23.07.2013) N4738'57.7" E16²8'16.7", 478 m. [8364.4]

7. Burgenland, Bezirk Oberpullendorf, Ritzing, by forest road (23.07.2013) N47 $38^{\prime} 57.7^{\prime \prime}$ E16²2'16.7.", 481 m. [8364.4]

8. Burgenland, Bezirk Oberpullendorf, Ritzing, in deciduous forest, by forest road (23.07.2013) N47³8'58.1" E16²7'59.0", 525 m. [8364.4]

9. Burgenland, Bezirk Oberpullendorf, Ritzing, in deciduous forest (23.07.2013) N47 $39^{\prime} 01.1^{\prime \prime}$ E16 $27^{\circ} 50.2^{\prime \prime}, 552 \mathrm{~m}$. [8364.2]

10. Burgenland, Bezirk Oberpullendorf, Ritzing, in deciduous forest, by forest road (23.07.2013) $\mathrm{N} 47^{\circ} 39^{\prime} 01.6^{\prime \prime} \mathrm{E} 16^{\circ} 27^{\prime} 37.8^{\prime \prime}, 544 \mathrm{~m}$. [8364.2]

11. Burgenland, Bezirk Oberpullendorf, Ritzing, by forest road (23.07.2013) N47 $39^{\prime} 04.2^{\prime \prime}$ E16 $27^{\circ} 37.0^{\prime \prime}, 543 \mathrm{~m}$. [8364.2]

12. Burgenland, Bezirk Oberpullendorf, Ritzing, on embankment of forest road (23.07.2013) N47³9'01.2" E16²7'32.9", 526 m. [8364.2]

13. Burgenland, Bezirk Oberpullendorf, Ritzing, in old Fagus sylvatica forest (23.07.2013) N4738'57.7" E16²27'33.2", 518 m. [8364.4]

14. Burgenland, Bezirk Oberpullendorf, Ritzing, near lake, by stream (23.07.2013) N47 38'55.20.0" E16 27'28.0", $494 \mathrm{~m}$. [8364.4]

15. Burgenland, Bezirk Oberpullendorf, Ritzing, by stream (23.07.2013) N47 $38^{\prime} 52.6^{\prime \prime}$ E16 27'30.9", 482 m. [8364.4]

16. Burgenland, Bezirk Oberpullendorf, Ritzing, dried puddles (23.07.2013) N47³8'41.0", E16²7'36.3", 467 m. [8364.4]

17. Burgenland, Bezirk Oberpullendorf, Ritzing, by forest road (23.07.2013) N47 $38^{\prime} 38.5^{\prime \prime}$, E16²7'41.7", 459 m. [8364.4]

18. Burgenland, Bezirk Oberpullendorf, Ritzing, grassland (23.07.2013) N47³8'30.8”, E16²7'58.2", 415 m. [8364.4]

19. Burgenland, Bezirk Oberpullendorf, Ritzing, in deciduous forest, by the forest road (23.07.2013) N4738'24.2", E16²8'6.4", 422 m. [8364.4]

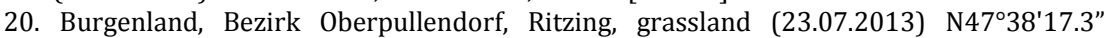
E16²28'10.6", 393 m. [8364.4]

21. Burgenland, Bezirk Oberpullendorf, Ritzing, Waldschule, by the road (23.07.2013) N4738'33.0" E16²8'32.4", 416 m. [8364.4]

22. Burgenland, Bezirk Oberpullendorf, Ritzing, old Fagus sylvatica forest, by the forest road (26.10.2013) N4738'45.8” E16²8'57.4", 482 m. [8364.4]

23. Burgenland, Bezirk Oberpullendorf, Ritzing, old Fagus sylvatica forest (26.10.2013) N47³8'42.7" E16²8'58.6", 491 m. [8364.4]

24. Burgenland, Bezirk Oberpullendorf, Ritzing, Lange Zeile, embankment of forest road (26.10.2013) N47³8'33.1" E16²8'59.0", 486 m. [8364.4]

25. Burgenland, Bezirk Oberpullendorf, Ritzing, Lange Zeile, crossroads (26.10.2013)

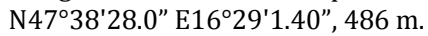


26. Burgenland, Bezirk Oberpullendorf, Ritzing, border of coniferous forest (26.10.2013) N4738' 25.6" E16²8'50.0", 481 m. [8364.4]

27. Burgenland, Bezirk Oberpullendorf, Ritzing, in coniferous forest (26.10.2013) N47³8'27.1" E16²8'41.1", 449 m. [8364.4]

28. Burgenland, Bezirk Oberpullendorf, Ritzing, in little valley (26.10.2013) N47 $38^{\prime} 31.2^{\prime \prime}$ E16²8'44.9", 434 m. [8364.4]

29. Burgenland, Bezirk Oberpullendorf, Ritzing, embankment of road, in wheel-tack (26.10.2013) N47³8'39.6”' E16²8'45.2", 432 m. [8364.4]

30. Burgenland, Bezirk Oberpullendorf, Ritzing, embankment of road, in wheel-tack (26.10.2013) N47'38'40.4" E16²8'46.5”, 435 m. [8364.4]

31. Burgenland, Bezirk Oberpullendorf, Ritzing, in old Fagus sylvatica forest (28.10.2013) N47³8'39.2" E16²9'5.1", 502 m. [8364.4]

32. Burgenland, Bezirk Oberpullendorf, Ritzing, in old Fagus sylvatica forest (28.10.2013) N47³8'30.7" E16²9'12.0", $512 \mathrm{~m}$. [8364.4]

33. Burgenland, Bezirk Oberpullendorf, Ritzing, in deciduous forest, embankment of forest road (28.10.2013) N47³8'9.1" E16 29'6.8”, 486 m. [8364.4]

34. Burgenland, Bezirk Oberpullendorf, Ritzing, in deciduous forest, embankment of forest

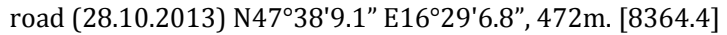

35. Burgenland, Bezirk Oberpullendorf, Ritzing, border of grassland (28.10.2013) N47³7'35.7" E16²9'25.5”, 379 m. [8364.4]

36. Burgenland, Bezirk Oberpullendorf, Ritzing, grassland (28.10.2013) N47³7'33.2" E16² 29'31.0", $370 \mathrm{~m}$. [8364.4]

37. Burgenland, Bezirk Oberpullendorf, Ritzing, grassland, stream (28.10.2013) N47 $37^{\prime} 50.4^{\prime \prime}$ E16²9'27.7”, 399 m. [8364.4]

38. Burgenland, Bezirk Oberpullendorf, Ritzing, in deciduous forest, embankment of forest

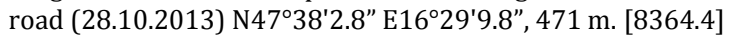

39. Burgenland, Bezirk Oberpullendorf, Ritzing, Pinus sylvestris forest, in glade (28.10.2013)

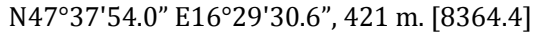

40. Burgenland, Bezirk Oberpullendorf, Ritzing, Pinus sylvestris forest, in glade (28.10.2013) N4737'54.1" E16²9'34.6", 444 m. [8364.4]

41. Burgenland, Bezirk Oberpullendorf, Ritzing, Pinus sylvestris forest, in glade (28.10.2013) $47^{\circ} 37^{\prime} 54.1^{\prime \prime} 16^{\circ} 29^{\prime} 34.5^{\prime \prime}, 444 \mathrm{~m}$. [8364.4]

42. Burgenland, Bezirk Oberpullendorf, Ritzing, Pinus sylvestris forest, in glade (28.10.2013) N473'ㄴ.2" E16²9'38.2", 456 m. [8364.4]

43. Burgenland, Bezirk Oberpullendorf, Ritzing, border of Pinus sylvestris forest, by forest road (28.10.2013) N47³7'53.0" E16²9'42.9”, 450 m. [8364.4]

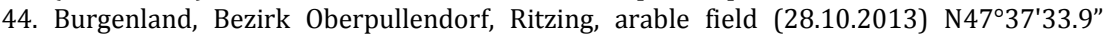
E16 29'58.2", 398 m. [8364.4]

45. Burgenland, Bezirk Oberpullendorf, Ritzing, arable field (28.10.2013) N47³7'29.9" E16 30'9.4", 395 m. [8365.3]

46. Burgenland, Bezirk Oberpullendorf, Ritzing, valley, by stream (28.10.2013) N47³7'39.1" E16²30'26.2", 367 m. [8365.3]

47. Burgenland, Bezirk Oberpullendorf, Ritzing, valley, by stream (28.10.2013) N47³7'40.4" E16 $30^{\circ} 24.1^{\prime \prime}, 368 \mathrm{~m}$. [8365.3]

48. Burgenland, Bezirk Oberpullendorf, Ritzing, gravelly road crossing (28.10.2013) $\mathrm{N} 47^{\circ} 37^{\prime} 43.5^{\prime \prime} \mathrm{E} 16^{\circ} 30^{\prime} 22.4^{\prime \prime}, 360 \mathrm{~m}$. [8365.3]

49. Burgenland, Bezirk Oberpullendorf, Ritzing, valley, by stream (28.10.2013) N47³7'44.9" E16 $30^{\prime} 17.4^{\prime \prime}, 371 \mathrm{~m}$. [8365.3]

50. Burgenland, Bezirk Oberpullendorf, Ritzing, valley, by stream (28.10.2013) N47³7'45.5" E16 $30^{\prime} 12.1^{\prime \prime}, 371 \mathrm{~m} .[8365.3]$

51. Burgenland, Bezirk Oberpullendorf, Ritzing, valley, by stream (28.10.2013) N47 $37^{\prime} 47.4^{\prime \prime}$ E16²30'10.4", 378 m. [8365.3]

52. Burgenland, Bezirk Oberpullendorf, Ritzing, valley, by stream (28.10.2013) N47³7'48.5" E16 30'08.6", 383 m. [8365.3] 
SzÚCS \& ZECHMEISTER (2016): Bryofloristical data from Austrian part of Sopron Hills

53. Burgenland, Bezirk Oberpullendorf, Ritzing, embankment of forest road (28.10.2013) N4737'53.3" E16²30'08.6", 401 m. [8365.3]

54. Burgenland, Bezirk Oberpullendorf, Ritzing, by forest road, little lake (28.10.2013) N473' $05.8^{\prime \prime}$ E16 $29^{\circ} 46.5^{\prime \prime}, 449$ m. [8364.4]

55. Burgenland, Bezirk Oberpullendorf, Ritzing, wet grassland (28.10.2013) N47 $37^{\prime} 59.0^{\prime \prime}$ E16 29'20.4", 418 m. [8364.4]

56. Burgenland, Bezirk Oberpullendorf, Ritzing, wet grassland (28.10.2013) N47 $38^{\prime} 00.4^{\prime \prime}$ E16 29'20.0", $421 \mathrm{~m}$. [8364.4]

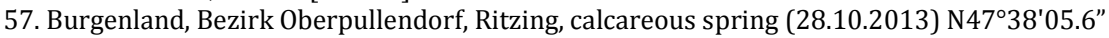
E16 $29 ' 18.6^{\prime \prime}, 447$ m. [8364.4]

58. Burgenland, Bezirk Oberpullendorf, Ritzing, by forest road (28.10.2013) N47³8'07.3" E16²9'19.9", 510 m. [8364.4]

59. Burgenland, Bezirk Oberpullendorf, Ritzing, Angerwald, near country border, deciduous forest (30.10.2013) N47³8'29.3" E16 $29^{\circ} 27.1^{\prime \prime}, 521 \mathrm{~m}$. [8364.4]

60. Burgenland, Bezirk Oberpullendorf, Ritzing, Angerwald, near country border, deciduous

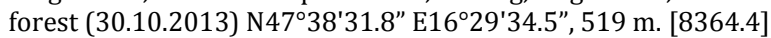

61. Burgenland, Bezirk Oberpullendorf, Ritzing, near country border, embankment of forest road (30.10.2013) N47³8'21.3" E16³0'15.9", 451 m. [8365.3]

62. Burgenland, Bezirk Oberpullendorf, Ritzing, Gruberkreuz, near country border, forest road crossing, little lake (30.10.2013) N47³8'16.9” E16 $30 ' 24.0^{\prime \prime}, 445 \mathrm{~m}$. [8365.3]

63. Burgenland, Bezirk Oberpullendorf, Ritzing, abandoned gneis-stone quarry $(30.10 .2013)$ $\mathrm{N} 47^{\circ} 38^{\prime} 35.5^{\prime \prime} \mathrm{E} 16^{\circ} 31^{\prime} 08.8^{\prime \prime}, 440 \mathrm{~m}$. [8365.3]

64. Burgenland, Bezirk Oberpullendorf, Ritzing, embankment of forest road (30.10.2013) N47³8'20.1" E16²31'23.8”, 445 m. [8365.3]

65. Burgenland, Bezirk Oberpullendorf, Ritzing, by forest road (30.10.2013) N47³7'59.4" E16 $31^{\prime} 40.0^{\prime \prime}, 345 \mathrm{~m}$. [8365.3]

66. Burgenland, Bezirk Oberpullendorf, Ritzing, embankment of forest road (30.10.2013) N47³7'58.2" E16²31'42.5”, 365 m. [8365.3]

67. Burgenland, Bezirk Oberpullendorf, Ritzing, embankment of forest road (30.10.2013) 47³7'34.6" E16 31'58.2", $330 \mathrm{~m}$. [8365.3]

68. Burgenland, Bezirk Oberpullendorf, Ritzing, by forest road (30.10.2013) N47³7'41.7" E16 $31^{\prime} 45.3 ", 340 \mathrm{~m}$. [8365.3]

69. Burgenland, Bezirk Oberpullendorf, Ritzing, abandoned quarry, recultivated places (30.10.2013) $\mathrm{N}^{\circ} 7^{\circ} 37^{\prime} 41.7^{\prime \prime} \mathrm{E} 16^{\circ} 31^{\prime} 45.3^{\prime \prime}, 340 \mathrm{~m}$. [8365.3]

70. Burgenland, Bezirk Oberpullendorf, Ritzing, embankment of forest road $(30.10 .2013)$ N47³7'30.1" E16³2'03.1", 325 m. [8365.3]

71. Burgenland, Bezirk Oberpullendorf, Neckenmarkt, fence of garden (30.10.2013) N47³7'10.6" E16²3'27.1", 290 m. [8365.3]

72. Burgenland, Bezirk Oberpullendorf, Neckenmarkt, weekend houses (30.10.2013) N47³7'13.1" E16²32'21.9", 300 m. [8365.3]

73. Burgenland, Bezirk Mattersburg, Marz, near recultivated quarry, by forest road (22.11.2013) N47² $42^{\prime} 16.8^{\prime \prime} \mathrm{E} 16^{\circ} 23^{\prime} 47.1^{\prime \prime}, 465 \mathrm{~m}$. [8264.3]

74. Burgenland, Bezirk Mattersburg, Sieggraben, by stream (22.11.2013) N4740'22.7" E16²3'54.6", $380 \mathrm{~m}$. [8364.1]

75. Burgenland, Bezirk Mattersburg, Sieggraben, wet grassland (22.11.2013) N47 $40^{\prime} 22.9^{\prime \prime}$ E16 $23^{\prime} 55.8^{\prime \prime}, 375$ m. [8364.1]

76. Burgenland, Bezirk Mattersburg, Sieggraben, wet grassland (22.11.2013) N47 $40 ' 22.4^{\prime \prime}$ E16²3'58.1", 385 m. [8364.1]

77. Burgenland, Bezirk Mattersburg, Sieggraben, wetland and stream (22.11.2013)

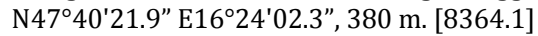

78. Burgenland, Bezirk Mattersburg, Sieggraben, wetland and stream (22.11.2013) N4740'20.7" E16²4'06.1", 385 m. [8364.1]

79. Burgenland, Bezirk Mattersburg, Sieggraben, embankment of forest (22.11.2013) N47²0'18.6" E16²4'03.6", 400 m. [8364.1] 
80. Burgenland, Bezirk Mattersburg, Sieggraben, by forest road, little pond (22.11.2013) N4740'18.8" E16²3'59.5", 395 m. [8364.1]

81. Burgenland, Bezirk Mattersburg, Sieggraben, near forest road (22.11.2013) N47²4'18.6" E16 24'03.6", 395 m. [8364.1]

82. Burgenland, Bezirk Mattersburg, Sieggraben, valley, by stream (22.11.2013) N47 $39^{\prime} 36.5^{\prime \prime}$ E16 22'53.1", 500 m. [8364.1]

83. Burgenland, Bezirk Mattersburg, Sieggraben, Satterhöhe, valley, by stream (22.11.2013) N4739'43.5" E16²2'49.3", 470 m. [8364.1]

84. Burgenland, Bezirk Mattersburg, Sieggraben, border of grassland, embankment of forest road (22.11.2013) N47³9'46.7" E16 $22^{\circ}$ '53.2", 455 m. [8364.1]

85. Burgenland, Bezirk Mattersburg, Sieggraben, valley, by sream (22.11.2013) N47³9'40.6" E16 22'52.9", 475 m. [8364.1]

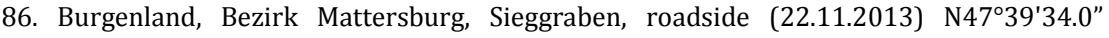
E16²2'45.1", 495 m. [8364.1]

87. Burgenland, Bezirk Mattersburg, Kalkgraben, grassland and roadside (22.11.2013) N4739'40.6" E16²2'52.9", 455 m. [8364.1]

88. Burgenland, Bezirk Mattersburg, Kalkgraben, grassland (22.11.2013) N47³9'40.6" E16 $22^{\prime} 52.9^{\prime \prime}, 420$ m. [8364.1]

89. Burgenland, Bezirk Oberpullendorf, Neckenmarkt, Millenium Teich, between two lakes (24.06.2014) N47³6'59.1" E16³4'05.0”, 235 m. [8365.3]

90. Burgenland, Bezirk Oberpullendorf, Neckenmarkt, embankment of forest road (24.06.2014) N47³7'33.2" E16³3’30.7”, 335 m. [8365.3]

91. Burgenland, Bezirk Oberpullendorf, Neckenmarkt, near embankment of forest road, deciduous forest (24.06.2014) N47³7'33.8” E16 33'27.4", 350 m. [8365.3]

92. Burgenland, Bezirk Oberpullendorf, Ritzing, in glade, grass, on soil (30.07.2014) N4739'07.6" E16²7'20.4", 545 m. [8364.2]

93. Burgenland, Bezirk Oberpullendorf, Ritzing, in mixed forest (30.07.2014) N47 $38^{\prime} 54.2^{\prime \prime}$ E16²7'03.6”, 565 m. [8364.4]

94. Burgenland, Bezirk Oberpullendorf, Ritzing, in mixed forest (30.07.2014) N47³8'46.5" E16 $26^{\prime} 56.4^{\prime \prime}, 545 \mathrm{~m}$. [8364.4]

95. Burgenland, Bezirk Oberpullendorf, Ritzing, in glade of planted pine forest, grass (30.07.2014) N47³8'20.1" E16 $26^{\prime} 43.2^{\prime \prime}, 440$ m. [8364.4]

96. Burgenland, Bezirk Oberpullendorf, Ritzing, next of planted pine forest, by forest road (30.07.2014) N47³8'15.5” E16²6’46.8”, 420 m. [8364.4]

97. Burgenland, Bezirk Oberpullendorf, Ritzing, by forest road (30.07.2014) N47 $38^{\prime} 10.2^{\prime \prime}$ E16 $26^{\prime} 41.7^{\prime \prime}, 405 \mathrm{~m}$. [8364.4]

98. Burgenland, Bezirk Oberpullendorf, Ritzing, wall of road cut (30.07.2014) N47³8'07.9” E16²6'43.7”, 420 m. [8364.4]

99. Burgenland, Bezirk Oberpullendorf, Ritzing, in coniferous forest (30.07.2014)

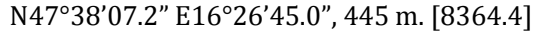

100. Burgenland, Bezirk Oberpullendorf, Ritzing, in coniferous forest (30.07.2014) N47³8'07.9" E16²6'42.8”, 445 m. [8364.4]

101. Burgenland, Bezirk Oberpullendorf, Ritzing, on embankment of forest road (30.07.2014) N47 $38^{\prime} 06.8^{\prime \prime} \mathrm{E} 16^{\circ} 26^{\prime} 36.9^{\prime \prime}, 410 \mathrm{~m}$. [8364.4]

102. Burgenland, Bezirk Oberpullendorf, Ritzing, by forest road (30.07.2014) N47 $38^{\prime} 06.1^{\prime \prime}$ E16 $26^{\prime} 35.4^{\prime \prime}, 410$ m. [8364.4]

103. Burgenland, Bezirk Oberpullendorf, Ritzing, on embankment of road (30.07.2014) N47³8'05.2" E16²6'35.3", 410 m. [8364.4]

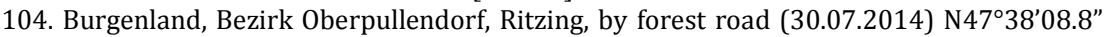
E16 26'33.6", 395 m. [8364.4]

105. Burgenland, Bezirk Oberpullendorf, Ritzing, embankment of forest road (30.07.2014) N47³8'10.1" E16²6'33.2", 405 m. [8364.4]

106. Burgenland, Bezirk Oberpullendorf, Ritzing, in Alnus glutinosa marsh (30.07.2014) N47³8'09.9” E16²6’31.8”, 400 m. [8364.4] 
107. Burgenland, Bezirk Oberpullendorf, Ritzing, by stream, water conversancy building (30.07.2014) N47³8'09.3" E16 $26^{\prime} 29.8^{\prime \prime}, 400$ m. [8364.4]

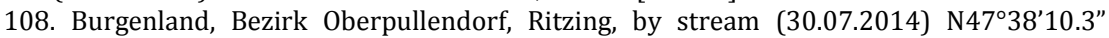
E16 26'29.3", 400 m. [8364.4]

109. Burgenland, Bezirk Oberpullendorf, Ritzing, in Alnus glutinosa marsh (30.07.2014) N47³8'10.8” E16²6'30.9”, 405 m. [8364.4]

110. Burgenland, Bezirk Oberpullendorf, Ritzing, by stream (30.07.2014) N47³8'11.6" E16 26'26.8”, 400 m. [8364.4]

111. Burgenland, Bezirk Oberpullendorf, Ritzing, by forest road (31.07.2014) N47³9'14.7" E16 $26^{\prime} 38.9^{\prime \prime}, 495$ m. [8364.2]

112. Burgenland, Bezirk Oberpullendorf, Ritzing, by forest road (31.07.2014) N47³9’04.7” E16 $26^{\prime} 38.1^{\prime \prime}, 480 \mathrm{~m}$. [8364.2]

113. Burgenland, Bezirk Oberpullendorf, Ritzing, by wet places (31.07.2014) N47³9'1.6" E16 26'26.6", 465 m. [8364.2]

114. Burgenland, Bezirk Oberpullendorf, Ritzing, near planted Picea abies forest, by forest road (31.07.2014) N47³8'49.9" E16 $26^{\prime} 04.1^{\prime \prime}, 460$ m. [8364.4]

115. Burgenland, Bezirk Oberpullendorf, Ritzing, by stream, on milestone (31.07.2014) N4738' 12.5" E16²6'25.6", 405 m. [8364.4]

116. Burgenland, Bezirk Oberpullendorf, Ritzing, by road-crossing, monument (31.07.2014) N4737'57.6" E16²6'21.3", 385 m. [8364.4]

117. Burgenland, Bezirk Oberpullendorf, Ritzing, in deciduous forest, coulloir (31.07.2014) N4738'41.2" E16²7'7.3", 525 m. [8364.4]

118. Burgenland, Bezirk Oberpullendorf, Ritzing, in deciduous forest, (31.07.2014) N47³8'40.1" E16² 27'4.8”, 515 m. [8364.4]

119. Burgenland, Bezirk Oberpullendorf, Ritzing, in deciduous forest, by forest road (31.07.2014) N47'38'39.9" E16 26'49.6", 530 m. [8364.4]

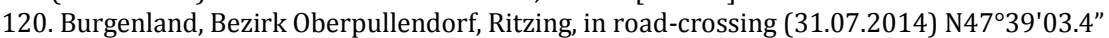
E16 $27^{\prime} 5.9^{\prime \prime}, 540 \mathrm{~m}$. [8364.2] 